\title{
Un-terminated, Low-Frequency Terminal-Behavioral $d-q$ Model of Three-Phase Converters
}

\author{
Igor Cvetkovic, Marko Jaksic, Dushan Boroyevich, Paolo Mattavelli, Fred C. Lee \\ Zhiyu Shen, Sara Ahmed and Dong Dong \\ Center for Power Electronics Systems (CPES) \\ The Bradley Department of Electrical and Computer Engineering \\ Virginia Tech, Blacksburg, VA 24061; USA; email: igorc@vt.edu
}

\begin{abstract}
Frequency-domain terminal-behavioral modeling of $a c$ systems is unquestionably attracting more and more interest in engineering practice. New electronic power distribution systems built for airplanes, ships, electric vehicles, data-centers and even homes, dominantly comprise a variety of power electronics converters with very different dynamic characteristics. If their behavior is not examined carefully before system is integrated, instability can become one of the major concerns. This paper addresses low-frequency terminal-behavioral modeling of three-phase converters which dynamics can be captured on-line, in a non-intrusive way, and later decoupled from the source and load in order to get un-terminated model of a particular converter (or a system). A few examples of different converters are given at the end to verify modeling and decoupling procedure with the average and switching models.
\end{abstract}

\section{INTRODUCTION}

Contemporary trends in building the future power distribution systems, some led by the sustainability concerns and some for pure economic reasons, open a space for high penetration of power electronics converters in electricity production, transmission, distribution and in general, utilization [1-5]. These systems consist of a wide assortment of different power converters, usually made by different manufacturers, and design of such systems entirely relies on the information provided in the datasheets and manuals, experience of system engineers, and their ability to test the system under different operating conditions. Thus, black-box modeling of converters can undoubtedly help with the system design and analysis prior to the integration.

Terminal-behavioral modeling of converters has been widely used in engineering practice for modeling and capturing the dynamic behavior of linear or "mildly" non-linear converters up to a half of the switching frequency. This modeling is very well understood and applied to $d c-d c$ converters [6-10] due to the easiness of performing small-signal measurements and existence of the operating point. This concept has even been further extended to address nonlinear behavior $[11,12]$ and EMI $[13,14]$. Although performing small-signal measurements on the $a c$ converters is not as straightforward due to the time varying steady-state, linearization of $a c$ networks around some desired operating point

This work was primarily supported by the CPES'

Renewable Energy and Microgrids mini-consortium can be done in rotating $d-q$ coordinates that are in synchronism with the input and/or output frequencies [15-18]. Smallsignal frequency-domain models can now be obtained by linearization around the desired operating point.

Although not directly applicable to the work presented in this paper, it should be mentioned that there are also the other methods to obtain small-signal models of $a c$ converters, such as modeling using phasors, reduced-order modeling and harmonic linearization [19].

This paper proposes the procedure for obtaining the unterminated terminal-behavioral model of power converters in a non-intrusive way; the small signal measurements can be obtained while converter is working on-line, without a need to be taken out of the system, characterized and put back. However, transfer functions obtained at the converter's terminals are terminated, meaning the dynamic properties of the source and load are inherently contained in the measured frequency response characteristics beside the converter's dynamics, and in order to obtain a good estimation of the converter's behavior, these dynamic properties of source and load side must be removed from the measured transfer functions. That procedure will be showed in the following sections of this paper.

\section{TERMINAL-BEHAVIORAL D-Q MODEL OF A THREE-PHASE CONVERTER}

Terminal-behavioral model of a three-phase converter can be represented as the four-port network shown in the Fig. 1. Its small-signal subsystem can be simply represented by the following expression:

$$
\left[\begin{array}{c}
\widetilde{\mathbf{v}}_{\mathrm{o}} \\
\widetilde{\mathbf{i}}_{\mathrm{i}}
\end{array}\right]=\left[\begin{array}{cc}
\mathbf{G}_{\mathrm{o}}(s) & -\mathbf{Z}_{\mathrm{o}}(s) \\
\mathbf{Y}_{\mathrm{i}}(s) & \mathbf{H}_{\mathrm{i}}(s)
\end{array}\right] \cdot\left[\begin{array}{c}
\widetilde{\mathbf{v}}_{\mathrm{i}} \\
\widetilde{\mathbf{i}}_{\mathrm{o}}
\end{array}\right]
$$

where

$$
\widetilde{\mathbf{v}}_{\mathrm{o}}=\left[\begin{array}{c}
\widetilde{v}_{o d} \\
\widetilde{v}_{o q}
\end{array}\right], \widetilde{\mathbf{v}}_{\mathrm{i}}=\left[\begin{array}{c}
\widetilde{v}_{i d} \\
\widetilde{v}_{i q}
\end{array}\right], \widetilde{\mathbf{i}}_{\mathrm{o}}=\left[\begin{array}{c}
\widetilde{i}_{o d} \\
\widetilde{i}_{o q}
\end{array}\right], \widetilde{\mathbf{i}}_{\mathrm{i}}=\left[\begin{array}{c}
\widetilde{i}_{i d} \\
\widetilde{i}_{i q}
\end{array}\right]
$$

are small-signal input and output voltages and currents, and 


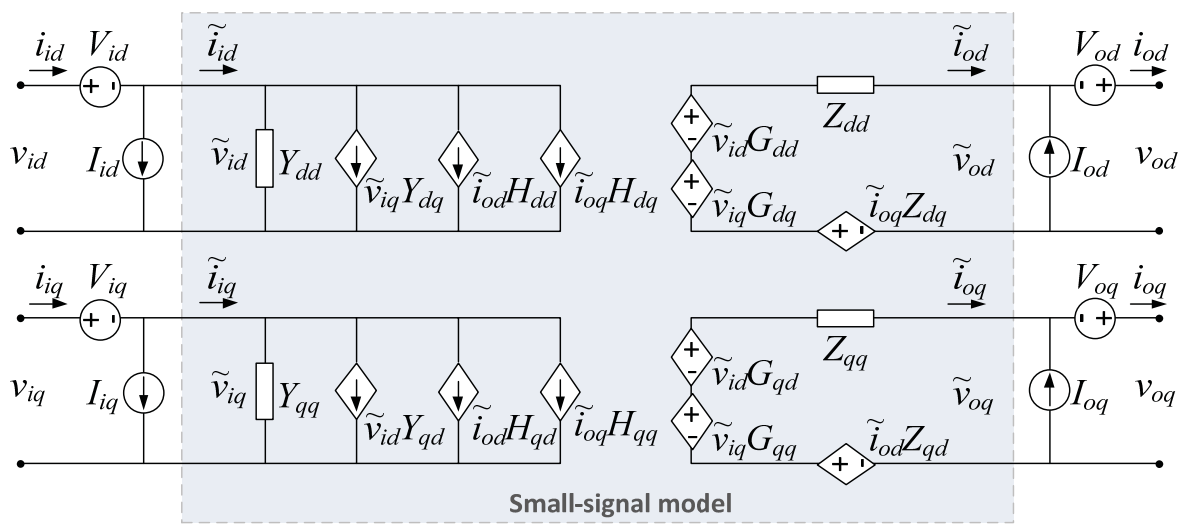

Figure 1. Modular terminal-behavioral model in synchronous $d-q$ frame of a generic balanced three-phase $a c$-ac converter

$$
\begin{aligned}
& \mathbf{G}_{\mathrm{o}}=\left[\begin{array}{ll}
G_{d d}(s) & G_{d q}(s) \\
G_{q d}(s) & G_{q q}(s)
\end{array}\right], \quad \mathbf{Z}_{\mathrm{o}}=\left[\begin{array}{cc}
Z_{d d}(s) & Z_{d q}(s) \\
Z_{q d}(s) & Z_{q q}(s)
\end{array}\right], \\
& \mathbf{Y}_{\mathrm{i}}=\left[\begin{array}{ll}
Y_{d d}(s) & Y_{d q}(s) \\
Y_{q d}(s) & Y_{q q}(s)
\end{array}\right], \quad \mathbf{H}_{\mathrm{i}}=\left[\begin{array}{cc}
H_{d d}(s) & H_{d q}(s) \\
H_{q d}(s) & H_{q q}(s)
\end{array}\right] ;
\end{aligned}
$$

are matrices of small-signal, un-terminated transfer functions, namely: $\mathbf{G}_{\mathrm{o}}$ - audio susceptibility, $\mathbf{Z}_{\mathrm{o}}$ - output impedance, $\mathbf{Y}_{\mathrm{i}}$ - input admittance and $\mathbf{H}_{\mathrm{i}}$ - back current gain.

To expand a system (1) into a system that includes an operating point around which the three-phase converter (network) is linearized, the form (5) has been obtained by using general expression (4) for all the variables.

$$
\begin{gathered}
x=X+\tilde{x} \\
{\left[\begin{array}{c}
\mathbf{v}_{\mathrm{o}} \\
\mathbf{i}_{\mathrm{i}}
\end{array}\right]=\left[\begin{array}{cc}
\mathbf{G}_{\mathrm{o}}(s)-\mathbf{Z}_{\mathrm{o}}(s) \\
\mathbf{Y}_{\mathrm{i}}(s) & \mathbf{H}_{\mathrm{i}}(s)
\end{array}\right] \cdot\left[\begin{array}{c}
\mathbf{V}_{\mathrm{i}}-\mathbf{V}_{\mathrm{i}} \\
\mathbf{i}_{\mathrm{o}}-\mathbf{I}_{\mathrm{o}}
\end{array}\right]+\left[\begin{array}{c}
\mathbf{V}_{\mathrm{o}} \\
\mathbf{I}_{\mathrm{i}}
\end{array}\right]}
\end{gathered}
$$

Variables (6) comprise both small-signal (2) and operating point values (7).

$$
\begin{gathered}
\mathbf{v}_{\mathrm{o}}=\left[\begin{array}{l}
v_{o d} \\
v_{o q}
\end{array}\right], \mathbf{v}_{\mathrm{i}}=\left[\begin{array}{c}
v_{i d} \\
v_{i q}
\end{array}\right], \mathbf{i}_{\mathrm{o}}=\left[\begin{array}{c}
i_{o d} \\
i_{o q}
\end{array}\right], \mathbf{i}_{\mathrm{i}}=\left[\begin{array}{c}
i_{i d} \\
i_{i q}
\end{array}\right] \\
\mathbf{V}_{\mathrm{o}}=\left[\begin{array}{c}
V_{o d} \\
V_{o q}
\end{array}\right], \mathbf{V}_{\mathrm{i}}=\left[\begin{array}{c}
V_{i d} \\
V_{i q}
\end{array}\right], \mathbf{I}_{\mathrm{o}}=\left[\begin{array}{c}
I_{o d} \\
I_{o q}
\end{array}\right], \mathbf{I}_{\mathrm{i}}=\left[\begin{array}{c}
I_{i d} \\
I_{i q}
\end{array}\right]
\end{gathered}
$$

Fig.1 is fully representing a terminal-behavioral model from (5).

\section{ObTAINING ThE TRANSFER FUnCtions}

As previously mentioned, this modeling requires transfer functions to be obtained in the $d-q$ coordinates. Although still under investigation by numerous researchers, some practical approaches have already been published on this subject [15-18]. Thus, let's consider the system shown in the Fig. 2 and assume that we are able to obtain small-signal variables (input and output currents and voltages in the $d-q$ domain) by using some of these methodologies. The transfer functions
(8-11) can be defined, with the subscript letter $m$ denoting "measured".

From the perturbation at point $\mathbf{I}$ ( $d$-axis, input side) (Fig. 2):

$$
G_{d d m}=\frac{\widetilde{v}_{o d}^{I}}{\widetilde{v}_{i d}^{I}}, G_{q d m}=\frac{\widetilde{v}_{o q}^{I}}{\widetilde{v}_{i d}^{I}}, Y_{d d m}=\frac{\tilde{i}_{i d}^{I}}{\widetilde{v}_{i d}^{I}}, Y_{q d m}=\frac{\widetilde{i}_{i q}^{I}}{\widetilde{v}_{i d}^{I}}
$$

from the perturbation at II ( $q$-axis, input side):

$$
G_{d q m}=\frac{\widetilde{v}_{o d}^{I I}}{\widetilde{v}_{i q}^{I I}}, G_{q q m}=\frac{\widetilde{v}_{o q}^{I I}}{\widetilde{v}_{i q}^{I I}}, Y_{d q m}=\frac{\widetilde{i}_{i d}^{I I}}{\widetilde{v}_{i q}^{I I}}, Y_{q q m}=\frac{\widetilde{i}_{i q}^{I I}}{\widetilde{v}_{i q}^{I I}} ;
$$

from the perturbation at III ( $d$-axis, output side):

$$
Z_{d d m}=-\frac{\widetilde{v}_{o d}^{I I I}}{\widetilde{i}_{o d}^{I I I}}, Z_{q d m}=-\frac{\widetilde{v}_{o q}^{I I I}}{\widetilde{i}_{o d}^{I I I}}, H_{d d m}=\frac{\widetilde{i}_{i d}^{I I I}}{\widetilde{i}_{o d}^{I I I}}, H_{q d m}=\frac{\widetilde{i}_{i q}^{I I I}}{\widetilde{i}_{o d}^{I I I}}
$$

from the perturbation at IV ( $q$-axis, output side):

$$
Z_{d q m}=-\frac{\widetilde{v}_{o d}^{I V}}{\widetilde{i}_{o q}^{I V}}, Z_{q q m}=-\frac{\widetilde{v}_{o q}^{I V}}{\widetilde{i}_{o q}^{I V}}, H_{d q m}=\frac{\widetilde{i}_{i d}^{I V}}{\widetilde{i}_{o q}^{I V}}, H_{q q m}=\frac{\widetilde{i}_{i q}^{I V}}{\widetilde{i}_{o q}^{I V}} ;
$$

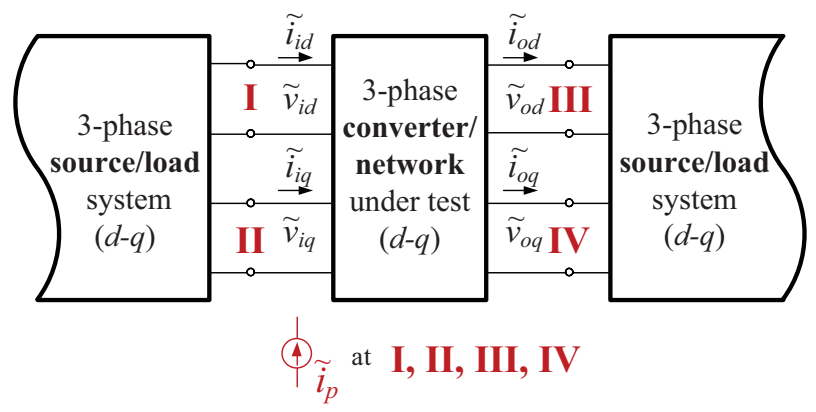

Figure. 2. Three-phase converter/network in $d-q$ frame 
It is highly important to repeat that above defined, measured transfer functions cannot be used to build the terminalbehavioral model (5) since they are terminated - comprise source and load dynamics. In order to obtain un-terminated transfer functions, the decoupling procedure has to be taken.

\section{TRANSFER FUNCTIONS DECOUPLING}

Let's now rewrite the small-signal model (1) in the following form:

$$
\begin{aligned}
& \tilde{v}_{o d}=G_{d d} \cdot \tilde{v}_{i d}+G_{d q} \cdot \tilde{v}_{i q}-Z_{d d} \cdot \tilde{i}_{o d}-Z_{d q} \cdot \tilde{i}_{o q} \\
& \tilde{v}_{o q}=G_{q d} \cdot \widetilde{v}_{i d}+G_{q q} \cdot \widetilde{v}_{i q}-Z_{q d} \cdot \widetilde{i}_{o d}-Z_{q q} \cdot \widetilde{i}_{o q} \\
& \widetilde{i}_{i d}=Y_{d d} \cdot \widetilde{v}_{i d}+Y_{d q} \cdot \widetilde{v}_{i q}+H_{d d} \cdot \widetilde{i}_{o d}+H_{d q} \cdot \widetilde{i}_{o q} \\
& \widetilde{i}_{i q}=Y_{q d} \cdot \widetilde{v}_{i d}+Y_{q q} \cdot \widetilde{v}_{i q}+H_{q d} \cdot \widetilde{i}_{o d}+H_{q q} \cdot \widetilde{i}_{o q}
\end{aligned}
$$

If we now take the transfer function $G_{d d m}$ from (8), rewrite it in the following form and combine with the first equation from (12), it can be written:

$$
\widetilde{v}_{o d}^{I}=G_{d d m} \cdot \widetilde{v}_{i d}^{I}
$$

and it must hold:

$$
\tilde{v}_{o d}^{I}=G_{d d m} \cdot \tilde{v}_{i d}^{I}=G_{d d} \cdot \tilde{v}_{i d}^{I}+G_{d q} \cdot \tilde{v}_{i q}^{I}-Z_{d d} \cdot \tilde{i}_{o d}^{I}-Z_{d q} \cdot \tilde{i}_{o q}^{I}
$$

Deriving (14) with $\tilde{v}_{i d}^{I}$ further simplifies the expression, and it can be obtain:

$$
G_{d d m}=G_{d d}+G_{d q} \cdot \frac{\widetilde{v}_{i q}^{I}}{\widetilde{v}_{i d}^{I}}-Z_{d d} \cdot \frac{\tilde{i}_{o d}^{I}}{\widetilde{v}_{i d}^{I}}-Z_{d q} \cdot \frac{\tilde{i}_{o q}^{I}}{\widetilde{v}_{i d}^{I}}
$$

This gives the first relationship between the unterminated and terminated transfer functions, but obviously cannot be solved directly since it has more unknowns than equations.

Continuing the same procedure for the next transfer function from the measurement point I - $G_{q d m}$, and combining it with the second equation from (12), it can be obtained:

$$
G_{q d m}=G_{q d}+G_{q q} \cdot \frac{\tilde{v}_{i q}^{I}}{\widetilde{v}_{i d}^{I}}-Z_{d q} \cdot \frac{\tilde{i}_{o d}^{I}}{\widetilde{v}_{i d}^{I}}-Z_{q q} \cdot \frac{\tilde{i}_{o q}^{I}}{\widetilde{v}_{i d}^{I}}
$$

Combining expression for $Y_{d d m}$ and the third equation from (12), it can be written:

$$
Y_{d d m}=Y_{d d}+Y_{q d} \cdot \frac{\widetilde{v}_{i q}^{I}}{\widetilde{v}_{i d}^{I}}+H_{d d} \cdot \frac{\widetilde{i}_{o d}^{I}}{\widetilde{v}_{i d}^{I}}+H_{d q} \cdot \frac{\tilde{i}_{o q}^{I}}{\widetilde{v}_{i d}^{I}}
$$

Finally, for the first set of measurements (point I), combining the $Y_{q d m}$ with the fourth equation from (12), expression (18) can be obtained.

$$
Y_{q d m}=Y_{q d}+Y_{q q} \cdot \frac{\tilde{v}_{i q}^{I}}{\widetilde{v}_{i d}^{I}}+H_{q d} \cdot \frac{\tilde{i}_{o d}^{I}}{\widetilde{v}_{i d}^{I}}+H_{q q} \cdot \frac{\tilde{i}_{o q}^{I}}{\widetilde{v}_{i d}^{I}}
$$

By looking at the previous four expressions (15-18), it can be noticed that all of them comprise three common transfer functions:

$$
\frac{\widetilde{v}_{i q}^{I}}{\widetilde{v}_{i d}^{I}}, \frac{\tilde{i}_{o d}^{I}}{\widetilde{v}_{i d}^{I}} \text { and } \frac{\widetilde{i}_{o q}^{I}}{\widetilde{v}_{i d}^{I}}
$$

Having the fact that all of the small-signal variables (input and output currents and voltages in both $d$ and $q$ axes) are already obtained from the measurement at point $\mathbf{I}$, these transfer functions can be considered known. The first one can be defined as a dimensionless transfer function $T V$, and the second two can be defined as $T G$ - transconductance due to the physical dimensions of the small-signal variable's units (output current over input voltage).

$$
T V_{q d m}=\frac{\widetilde{v}_{i q}^{I}}{\widetilde{v}_{i d}^{I}}, T G_{d d m}=\frac{\tilde{i}_{o d}^{I}}{\widetilde{v}_{i d}^{I}} \text { and } T G_{q d m}=\frac{\tilde{i}_{o q}^{I}}{\widetilde{v}_{i d}^{I}}
$$

The system (15-18) can now be rewritten as:

$$
\begin{aligned}
& G_{d d m}=G_{d d}+G_{d q} \cdot T V_{q d m}-Z_{d d} \cdot T G_{d d m}-Z_{d q} \cdot T G_{q d m} \\
& G_{q d m}=G_{q d}+G_{q q} \cdot T V_{q d m}-Z_{q d} \cdot T G_{d d m}-Z_{q q} \cdot T G_{q d m} \\
& Y_{d d m}=Y_{d d}+Y_{d q} \cdot T V_{q d m}+H_{d d} \cdot T G_{d d m}+H_{d q} \cdot T G_{q d m} \\
& Y_{q d m}=Y_{q d}+Y_{q q} \cdot T V_{q d m}+H_{q d} \cdot T G_{d d m}+H_{q q} \cdot T G_{q d m}
\end{aligned}
$$

The system has now four equation but sixteen unknowns, thus still cannot be solved for the un-terminated transfer functions.

All given above starting from (13) was used to build the system of equations for the transfer functions obtained by perturbing the system on the input (at $d$ axis - point I). Following the same procedure for the measurements at points II, III and IV, below shown results can be obtained.

System of transfer functions obtained from the perturbation at the point II:

$$
\begin{aligned}
& G_{d q m}=G_{d d} \cdot T V_{d q m}+G_{d q}-Z_{d d} \cdot T G_{d q m}-Z_{d q} \cdot T G_{q q m} \\
& G_{q q m}=G_{q d} \cdot T V_{d q m}+G_{q q}-Z_{q d} \cdot T G_{d q m}-Z_{q q} \cdot T G_{q q m} \\
& Y_{d q m}=Y_{d d} \cdot T V_{d q m}+Y_{d q}+H_{d d} \cdot T G_{d q m}+H_{d q} \cdot T G_{q q m} \\
& Y_{q d m}=Y_{q d} \cdot T V_{d q m}+Y_{q q}+H_{q d} \cdot T G_{d q m}+H_{q q} \cdot T G_{q q m}
\end{aligned}
$$

Similarly as above, for this set of equations, the following transfer functions have to be defined:

$$
T V_{d q m}=\frac{\widetilde{v}_{i d}^{I I}}{\widetilde{v}_{i q}^{I I}}, T G_{d q m}=\frac{\widetilde{i}_{o d}^{I I}}{\widetilde{v}_{i q}^{I I}} \text { and } T G_{q q m}=\frac{\widetilde{i}_{o q}^{I I}}{\widetilde{v}_{i q}^{I I}}
$$

System of transfer functions obtained from the perturbation at the point III:

$$
\begin{aligned}
& -Z_{d d m}=G_{d d} \cdot T R_{d d m}+G_{d q} \cdot T R_{q d m}-Z_{d d}-Z_{d q} \cdot T I_{q d m} \\
& -Z_{q d m}=G_{q d} \cdot T R_{d d m}+G_{q q} \cdot T R_{q d m}-Z_{q d}-Z_{q q} \cdot T I_{q d m} \\
& H_{d d m}=Y_{d d} \cdot T R_{d d m}+Y_{d q} \cdot T R_{q d m}+H_{d d}+H_{d q} \cdot T I_{q d m} \\
& H_{q d m}=Y_{q d} \cdot T R_{d d m}+Y_{q q} \cdot T R_{q d m}+H_{q d}+H_{q q} \cdot T I_{q d m}
\end{aligned}
$$

The system (24) comprises a few new transfer functions to be defined: the first two can be defined as TR-transconductance due to the physical dimensions of the smallsignal variable's units (input voltage over output current), and the third one as dimensionless transfer function $T I$ : 


$$
T R_{d d m}=\frac{\widetilde{v}_{i d}^{I I I}}{\widetilde{i}_{o d}^{I I I}}, T R_{q d m}=\frac{\widetilde{v}_{i q}^{I I I}}{\widetilde{i}_{o d}^{I I I}} \text { and } T I_{q d m}=\frac{\widetilde{i}_{o q}^{I I I}}{\widetilde{i}_{o d}^{I I I}}
$$

Finally, system of transfer functions obtained from the perturbation at the point IV:

$$
\begin{aligned}
& -Z_{d q m}=G_{d d} \cdot T R_{d q m}+G_{d q} \cdot T R_{q q m}-Z_{d d} \cdot T I_{d q m}-Z_{d q} \\
& -Z_{q q m}=G_{q d} \cdot T R_{d q m}+G_{q q} \cdot T R_{q q m}-Z_{q d} \cdot T I_{d q m}-Z_{q q} \\
& H_{d q m}=Y_{d d} \cdot T R_{d q m}+Y_{d q} \cdot T R_{q q m}+H_{d d} \cdot T I_{d q m}+H_{d q} \\
& H_{q q m}=Y_{q d} \cdot T R_{d q m}+Y_{d q} \cdot T R_{q q m}+H_{q d} \cdot T I_{d q m}+H_{q q}
\end{aligned}
$$

For this set of equations, the following transfer functions have to be defined:

$$
T R_{d q m}=\frac{\widetilde{v}_{i d}^{V I}}{\widetilde{i}_{o q}^{V I}}, T R_{q q m}=\frac{\widetilde{v}_{i q}^{V I}}{\widetilde{i}_{o q}^{V I}} \text { and } T I_{q d m}=\frac{\widetilde{i}_{o d}^{I V}}{\widetilde{i}_{o q}^{I V}}
$$

At last, the system of sixteen equations (21), (22), (24), (26), and sixteen unknowns has been built, and can be solved for un-terminated transfer functions. The solution is shown in (28).

$$
\left[\begin{array}{cc}
\mathbf{G}_{\mathrm{o}} & -\mathbf{Z}_{\mathrm{o}} \\
\mathbf{Y}_{\mathrm{i}} & \mathbf{H}_{\mathrm{i}}
\end{array}\right]=\left[\begin{array}{cc}
\mathbf{G}_{\mathrm{om}} & -\mathbf{Z}_{\mathrm{om}} \\
\mathbf{Y}_{\mathrm{im}} & \mathbf{H}_{\mathrm{im}}
\end{array}\right] \cdot\left[\mathbf{T}^{\mathrm{ac}-\mathrm{ac}}\right]^{-1}
$$

where

$$
\mathbf{T}^{\mathrm{ac}-\mathrm{ac}}=\left[\begin{array}{cccc}
1 & T V_{d q m} & T R_{d d m} & T R_{d q m} \\
T V_{q d m} & 1 & T R_{q d m} & T R_{q q m} \\
T G_{d d m} & T G_{d q m} & 1 & T I_{d q m} \\
T G_{q d m} & T G_{q q m} & T I_{q d m} & 1
\end{array}\right]
$$

Is the transfer functions decoupling matrix with the previously defined elements (20), (23), (25) and (27).

\section{Terminal-Behavioral Models of AC-DC, DC-AC AND DC-DC CONVERTERS}

System in (5) actually represents the generic terminalbehavioral model in $d$ - $q$ coordinates of all four types of converters (networks) $a c-a c, a c-d c, d c-a c$ and $d c-d c$. The only difference will be the number of variables and transfer functions that describe particular converter type.

ac-dc converter variables, transfer functions and decoupling matrix:

$$
\begin{aligned}
& \mathbf{v}_{\mathrm{o}}=\left[v_{o d}\right], \mathbf{v}_{\mathrm{i}}=\left[\begin{array}{c}
v_{i d} \\
v_{i q}
\end{array}\right], \mathbf{i}_{\mathrm{o}}=\left[i_{o d}\right], \mathbf{i}_{\mathrm{i}}=\left[\begin{array}{c}
i_{i d} \\
i_{i q}
\end{array}\right] \\
& \mathbf{V}_{\mathrm{o}}=\left[V_{o d}\right], \mathbf{V}_{\mathrm{i}}=\left[\begin{array}{c}
V_{i d} \\
V_{i q}
\end{array}\right], \mathbf{I}_{\mathrm{o}}=\left[I_{o d}\right], \mathbf{I}_{\mathrm{i}}=\left[\begin{array}{c}
I_{i d} \\
I_{i q}
\end{array}\right]
\end{aligned}
$$

$$
\begin{aligned}
& \mathbf{G}_{\mathrm{o}}^{\mathrm{ac}-\mathrm{dc}}=\left[\begin{array}{ll}
G_{d d} & G_{d q}
\end{array}\right], \quad \mathbf{Z}_{\mathrm{o}}^{\mathrm{ac}-\mathrm{dc}}=\left[Z_{d d}\right], \\
& \mathbf{Y}_{\mathrm{i}}^{\mathrm{ac}-\mathrm{dc}}=\left[\begin{array}{ll}
Y_{d d} & Y_{d q} \\
Y_{q d} & Y_{q q}
\end{array}\right], \quad \mathbf{H}_{\mathrm{i}}^{\mathrm{ac}-\mathrm{dc}}=\left[\begin{array}{c}
H_{d d} \\
H_{q d}
\end{array}\right] ;
\end{aligned}
$$

$$
\mathbf{T}^{\mathrm{ac}-\mathrm{dc}}=\left[\begin{array}{ccc}
1 & T V_{d q m} & T R_{d d m} \\
T V_{q d m} & 1 & T R_{q d m} \\
T G_{d d m} & T G_{d q m} & 1
\end{array}\right]
$$

$\boldsymbol{d c}$-ac converter variables, transfer functions and decoupling matrix:

$$
\begin{aligned}
& \mathbf{v}_{\mathrm{o}}=\left[\begin{array}{l}
v_{o d} \\
v_{o q}
\end{array}\right], \mathbf{v}_{\mathrm{i}}=\left[v_{i d}\right], \mathbf{i}_{\mathrm{o}}=\left[\begin{array}{c}
i_{o d} \\
i_{o q}
\end{array}\right], \mathbf{i}_{\mathrm{i}}=\left[i_{i d}\right] \\
& \mathbf{V}_{\mathrm{o}}=\left[\begin{array}{c}
V_{o d} \\
V_{o q}
\end{array}\right], \mathbf{V}_{\mathrm{i}}=\left[V_{i d}\right], \mathbf{I}_{\mathrm{o}}=\left[\begin{array}{c}
I_{o d} \\
I_{o q}
\end{array}\right], \mathbf{I}_{\mathrm{i}}=\left[I_{i d}\right] \\
& \mathbf{G}_{\mathrm{o}}^{\mathrm{dc}-\mathrm{ac}}=\left[\begin{array}{l}
G_{d d} \\
G_{q d}
\end{array}\right], \quad \mathbf{Z}_{\mathrm{o}}^{\mathrm{dc}-\mathrm{ac}}=\left[\begin{array}{ll}
Z_{d d} & Z_{d q} \\
Z_{q d} & Z_{q q}
\end{array}\right], \\
& \mathbf{Y}_{\mathrm{i}}^{\mathrm{dc}-\mathrm{ac}}=\left[Y_{d d}\right], \quad \mathbf{H}_{\mathrm{i}}^{\mathrm{dc}-\mathrm{ac}}=\left[\begin{array}{ll}
H_{d d} & H_{d q}
\end{array}\right] ; \\
& \mathbf{T}^{\mathrm{dc}-\mathrm{ac}}=\left[\begin{array}{ccc}
1 & T R_{d d m} & T R_{d q m} \\
T G_{d d m} & 1 & T I_{d q m} \\
T G_{q d m} & T I_{q d m} & 1
\end{array}\right]
\end{aligned}
$$

$d c$-dc converter variables, transfer functions and decoupling matrix:

$$
\begin{gathered}
\mathbf{v}_{\mathrm{o}}=\left[v_{o d}\right], \mathbf{v}_{\mathrm{i}}=\left[v_{i d}\right], \mathbf{i}_{\mathrm{o}}=\left[i_{o d}\right], \mathbf{i}_{\mathrm{i}}=\left[i_{i d}\right] \\
\mathbf{V}_{\mathrm{o}}=\left[V_{o d}\right], \mathbf{V}_{\mathrm{i}}=\left[V_{i d}\right], \mathbf{I}_{\mathrm{o}}=\left[I_{o d}\right], \mathbf{I}_{\mathrm{i}}=\left[I_{i d}\right] \\
\mathbf{G}_{\mathrm{o}}^{\mathrm{dc}-\mathrm{dc}}=\left[G_{d d}\right], \quad \mathbf{Z}_{\mathrm{o}}^{\mathrm{dc}-\mathrm{dc}}=\left[Z_{d d}\right], \\
\mathbf{Y}_{\mathrm{i}}^{\mathrm{dc}-\mathrm{dc}}=\left[Y_{d d}\right], \quad \mathbf{H}_{\mathrm{i}}^{\mathrm{dc}-\mathrm{dc}}=\left[H_{d d}\right] ; \\
\mathbf{T}^{\mathrm{dc}-\mathrm{dc}}=\left[\begin{array}{cc}
1 & T R_{d d m} \\
T G_{d d m} & 1
\end{array}\right]
\end{gathered}
$$

Finally, generalized expression for obtaining the unterminated transfer functions for any of the $a c-a c, d c-a c, a c-$ $d c$ and $d c-d c$ converters (or, in general, for any two- or fourport network) is given in (42). 


$$
\left[\begin{array}{cc}
\mathbf{G}_{\mathrm{o}}^{\mathrm{x}} & -\mathbf{Z}_{\mathrm{o}}^{\mathrm{x}} \\
\mathbf{Y}_{\mathrm{i}}^{\mathrm{x}} & \mathbf{H}_{\mathrm{i}}^{\mathrm{x}}
\end{array}\right]=\left[\begin{array}{cc}
\mathbf{G}_{\mathrm{om}}^{\mathrm{x}} & -\mathbf{Z}_{\mathrm{om}}^{\mathrm{x}} \\
\mathbf{Y}_{\mathrm{im}}^{\mathrm{x}} & \mathbf{H}_{\mathrm{im}}^{\mathrm{x}}
\end{array}\right] \cdot\left[\mathbf{T}^{\mathrm{x}}\right]^{-1}
$$

where $\mathrm{x}=\{\mathrm{ac}-\mathrm{ac}, \mathrm{ac}-\mathrm{dc}, \mathrm{dc}-\mathrm{ac}, \mathrm{dc}-\mathrm{dc}\}$

\section{Verification of the Decoupling Procedure}

\section{Linear network}

In order to verify the decoupling procedure, three-phase, balanced linear network with non-ideal source and load was used as shown in the Fig. 3a. All parameters are given, and circuit is shown in both $a b c$ and $d-q$ domain.

Small-signal input and output currents and voltages in $d$ and $q$ axes have been obtained by performing the linearization (linear analysis) of the circuit in Matlab, around the desired operating point, at the terminals I, II, III and IV as shown in Fig. 3a. Applying the above given definitions: (8), (9), (10), (11), (20), (23), (25) and (27), 28 transfer functions are obtained.

To verify the result of the decoupling procedure, un-terminated transfer functions of the linear network from Fig. 3a are analytically derived (with no source and load connected) showing matching with the ones obtained through the circuit linearization and decoupling (42). As an illustration, output impedance in all three cases: terminated, un-terminated and analytically obtained are all overplotted and shown in the Fig. 3b.

Analytical expression for the output impedance $Z_{d d}$ used above is shown in (43).

$$
\begin{aligned}
& Z_{\text {odd }}= \\
& \frac{L R\left(s\left(R+L s+C L R s^{2}\right)+L(1+C R s) \omega^{2}\right)}{\left(R+L s+C L R s^{2}\right)^{2}+L \omega^{2}\left(L(1+2 C R s(1+C R s))-2 C R^{2}\right)+C^{2} L^{2} R^{2} \omega^{4}}
\end{aligned}
$$

Moreover, the time domain responses of the circuit shown in the Fig. 3a were captured when the load step was
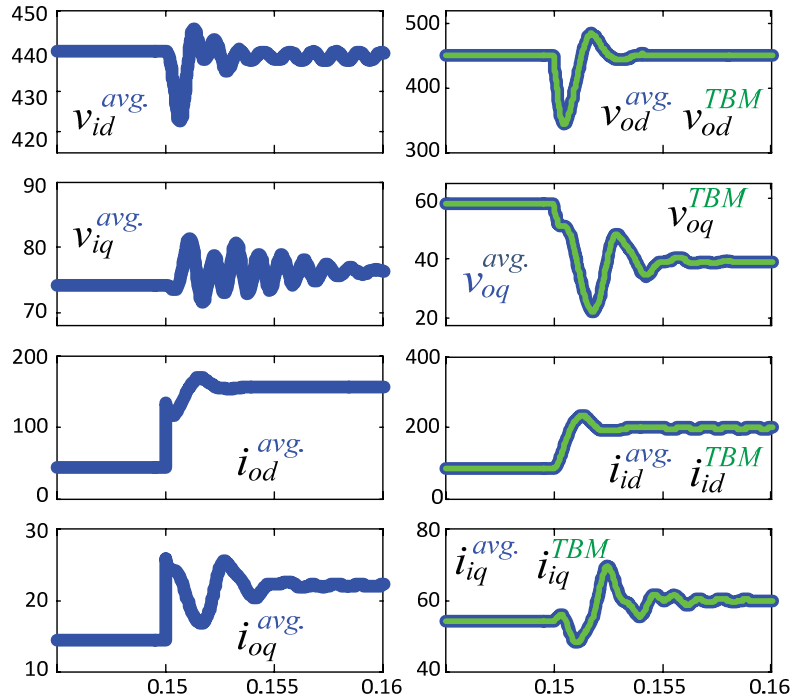

Figure 4. Time domain responses in $d$ and $q$ axes for the given load step, and comparison with the terminal-behavioral model (TBM).

applied (at $0.15 \mathrm{~s}, 4 \Omega$ resistors were added in parallel to the $10 \Omega$ ones). Transient responses of all eight variables $\left(v_{i d}, v_{i q}\right.$, $i_{o d}, i_{o q}, v_{o d}, v_{o q}, i_{i d}$ and $\left.i_{i q}\right)$ are shown in the Fig. 4 colored in blue. The system was now modeled in frequency domain using Terminal-Behavioral Model (TBM) (5) and transfer functions obtained through the linear analysis in Matlab as mentioned earlier in this section. As it can be noticed in model (5), input variables to the system (in $d-q$ axes) are input voltages and output currents, whereas the output variables are output voltages and input currents. In order to validate our modeling procedure, by applying the waveforms shown on the left side of the Fig. 4 (input variables for the model), to the model (5), its output must give very similar response (waveforms) as the recorder output voltage and input currents (from an average model) in order to claim

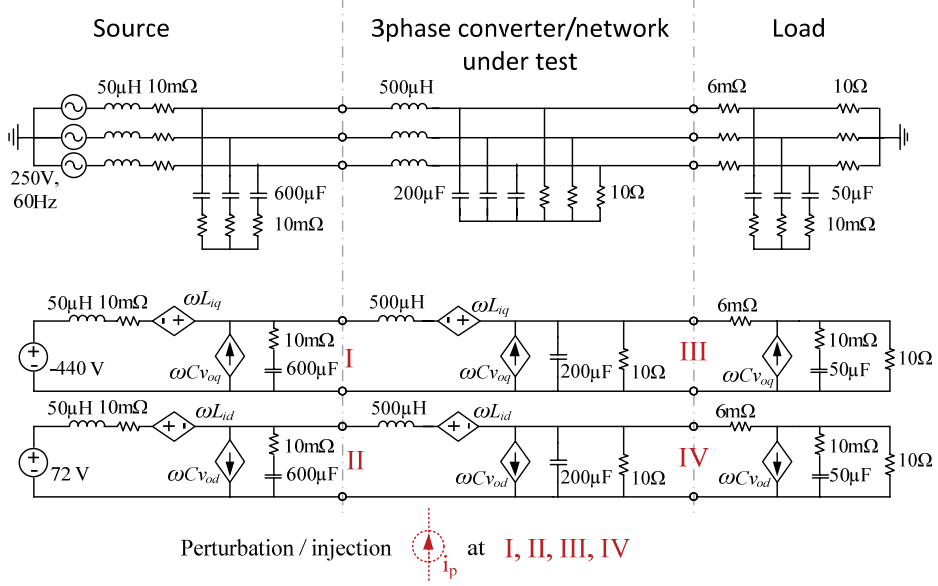

a)

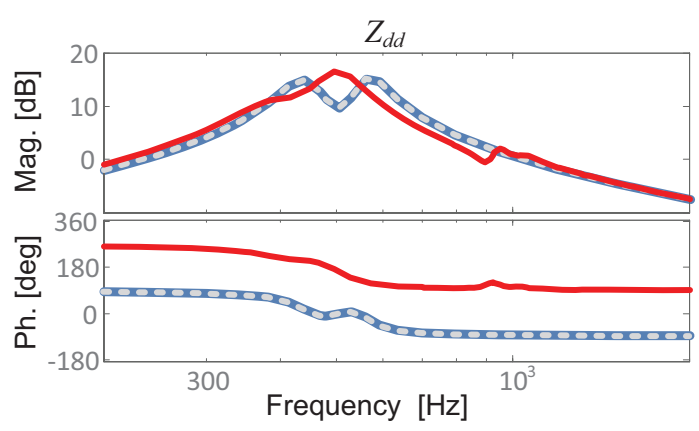

b)

Figure 3. a) Three phase source, linear $R-L-C$ network and a load b) Output impedance $Z_{d d}$, red - before decoupling, terminated, blue - after decoupling (using (42), and gray dotted - analytically obtained and overplotted for the comparison 
that the model is good. These, model output responses were overplotted over the voltages and currents shown on the right side of the Fig. 4, and as evident, a good matching has been obtained.

\section{$A C$-AC Converter}

Another example used for the verification purpose is an average model of the $a c$ - $a c$ converter (back-to-back converter) shown in the Fig.5a. All of the circuit parameters are given in the same figure. Source and a load used in this model were the same as the ones used in the example of the linear network shown in Fig. 3a.

The procedure for obtaining the transfer functions and building the terminal-behavioral model was identical as shown above in the linear network case. Although total of sixteen un-terminated transfer functions was obtained, only four of them are shown below in the Fig. 5b. in order to conserve space. Shown are terminated and un-terminated $G_{d d}, Z_{d d}, Y_{d d}$ and $H_{d d}$ emphasizing the influence of the source

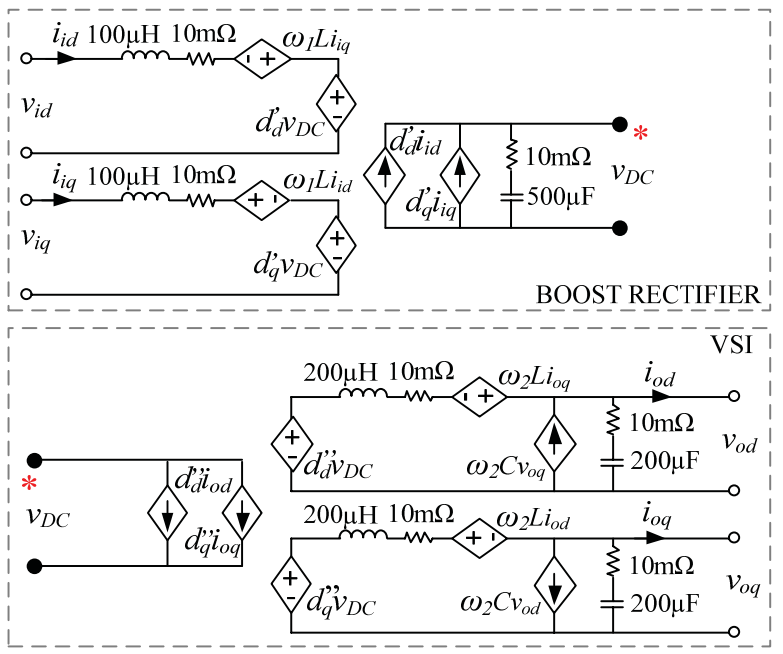

a)

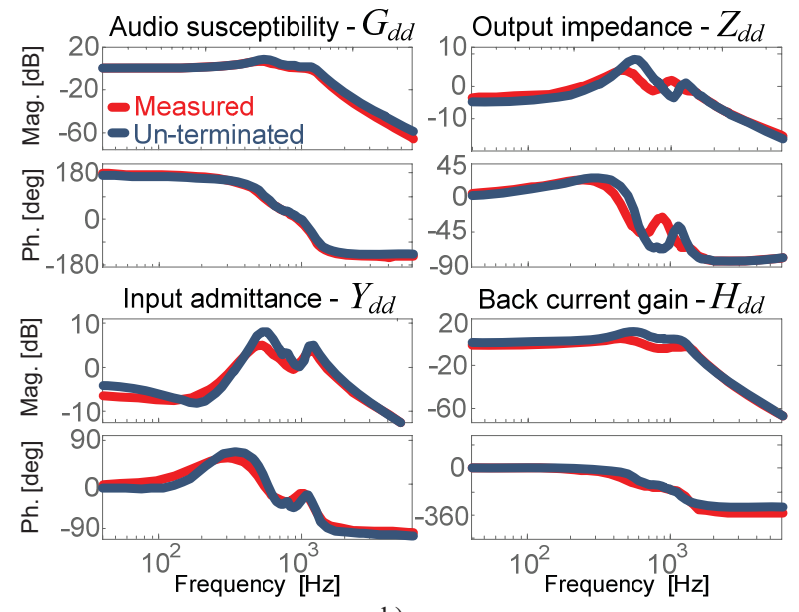

b)

Figure 5. a) Average model of $a c-a c$ converter in $d-q$, b) transfer functions: red - before decoupling, terminated, blue - after decoupling
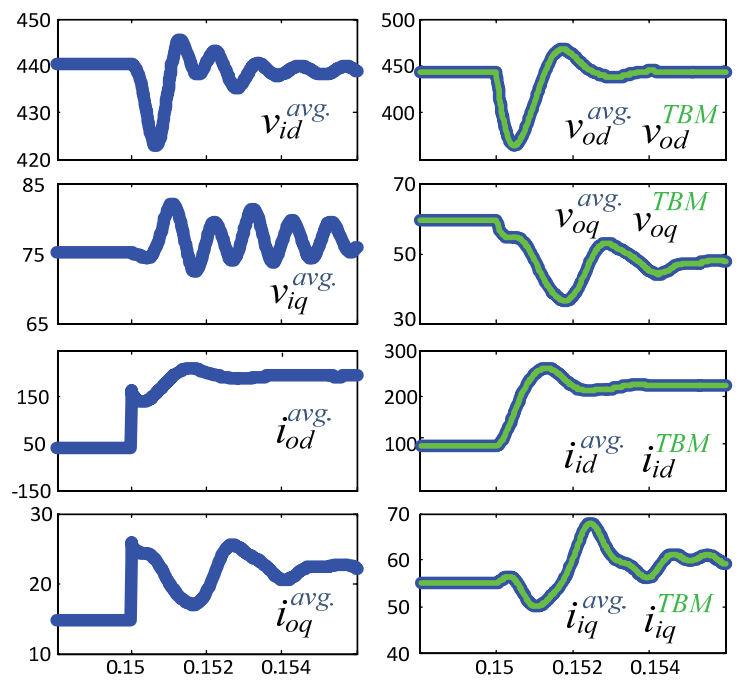

Figure 6. Time domain responses in $d$ and $q$ axes for the given load step, and comparison with the terminal-behavioral model (TBM).

and load dynamics that was taken out by applying decoupling procedure (42).

Finally, the time domain dynamic response was captured from the average model simulation and shown in the Fig. 6 together with the frequency domain model results (5). Again, very good matching between two models was achieved.

\section{$A C-D C$ Converter}

The third example used for verification was a switching model of a regulated Active Front End (AFE) shown in the Fig. 7 with all the circuit parameters. As a source, three-
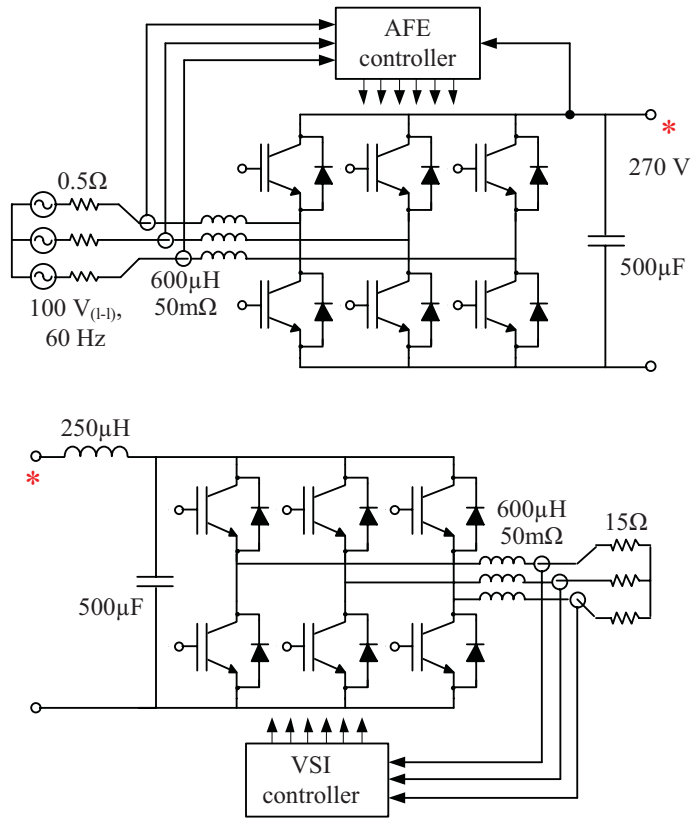

Figure 7. Active Front End and Voltage Source Inverter 


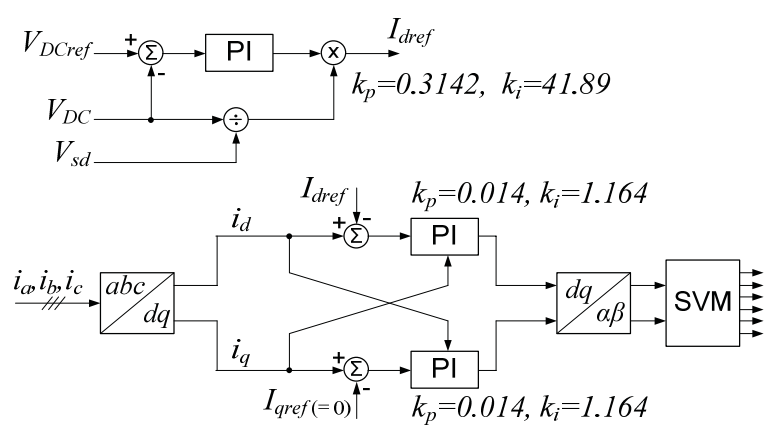

Fig. 8. Voltage controller of the AFE, and current controller of both AFE and VSI

phase non-ideal source was used, while regulated Voltage Source Inverter (VSI) loaded with resistors was used as a load. AFE had voltage and current loop closed, while VSI featured current loop only. Control parameters for both AFE and VSI are shown in the Fig. 8 (both converters had, for simplicity, the same control parameters for current loops, and both were switching at $20 \mathrm{kHz}$ ).

The $a c$ analysis was performed over the switching model of AFE circuit using Matlab. For each of the small-signal variables $\left(v_{i d}, v_{i q}, i_{o d}, i_{o q}, v_{o d}, v_{o q}, i_{i d}\right.$ and $\left.i_{i q}\right)$ obtained were gains and phases at 31 frequency points in the range from $100 \mathrm{~Hz}$ to $10 \mathrm{kHz}$. Applying the above given definitions: (8), (9), (10), (11), (20), (23), (25) and (27), total of 28 transfer functions was obtained in the vector form with complex numbers - frequency response data. This is shown in the Fig. 9 with the yellow dots for the four transfer functions $G_{d d}, Z_{d d}, Y_{d d}$ and $H_{d d}$. Due to the fact that terminalbehavioral model (5) cannot be built with the frequency response data, all transfer functions had to be identified in the $s$-domain using some identification tool. Function 'fitfrd' was used here (Matlab) to perform identification of the frequency response data, and the red colored transfer functions from Fig. 9 were obtained. Afterward, the expression (42) was used to decouple the source and load dynamics from the converter's dynamics. The blue colored unterminated transfer functions (in $d d$ ) are shown in the Fig. 9 plotted behind the terminated ones.

Lastly, the load step was applied on the circuit (the current reference of the VSI was stepped up from 5A to 7A at $0.5 \mathrm{~s}$ in order to simulate the load change), and time domain waveforms have been obtained. Fig. 10 shows the time domain response of the three variables that are acquired as output variables from the terminal-behavioral model (5) output votage $v_{o d}$, input current $i_{i d}$ in $d$-axis, and input current $i_{i q}$ in $q$-axis. For the comparison purposes, the same system (from Fig. 7) has been modeled in an average model as well as in the switching, and results in the time domain are shown for all three of them: switching model, average model and terminal-behavioral model. It can be seen and concluded that terminal-behavioral model (being a lowfrequency model) nicely tracks dynamics of the recorded variables, with a small steady state error after the transient settles down, which is due to the non-linear control (Fig. 8), but also due to the non-linear nature of the boost converter.

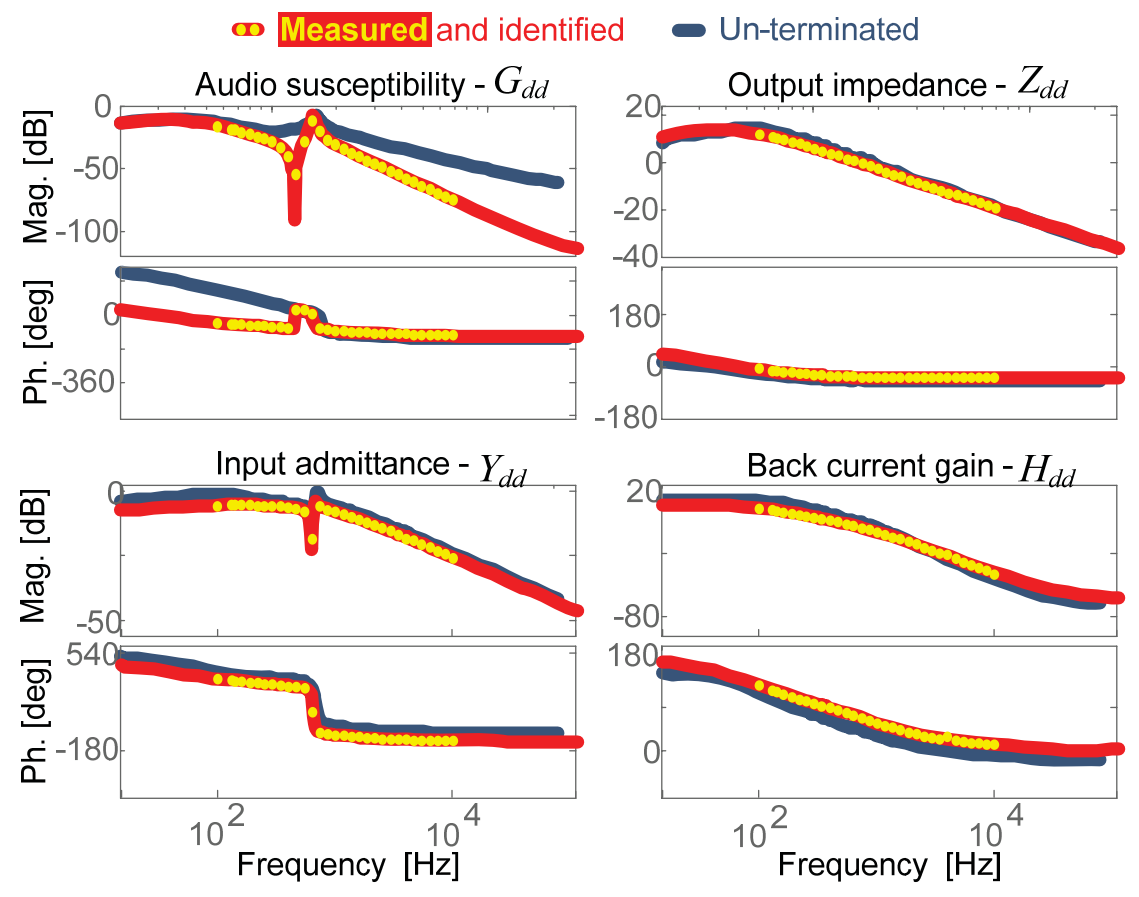

Figure 9. Transfer functions obtained from the switching model: yellow-measured points, red - transfer functions identified in the s-domain from the measured points (before decoupling)- terminated, blue - after decoupling (using (42)) 

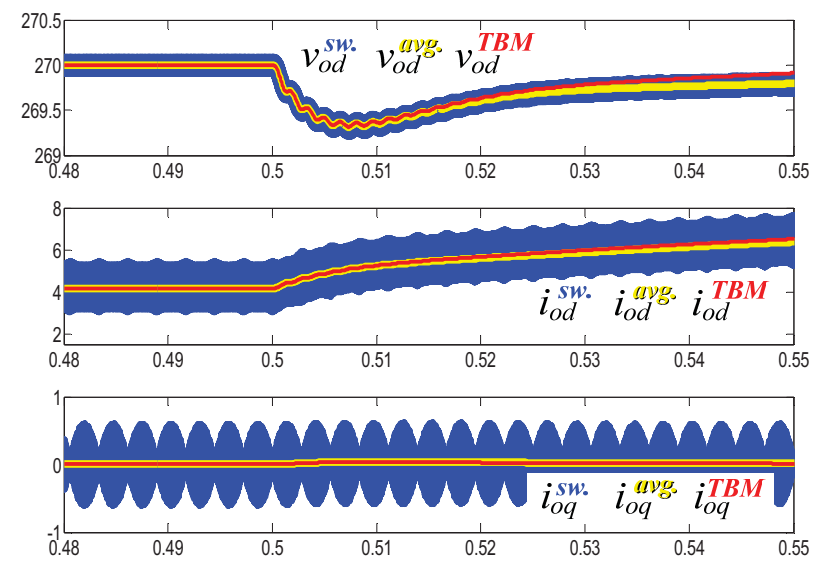

Figure 10. Time domain responses in $d$ and $q$ axes for the given load step, and comparison of a switching (sw.), average (avg.) and terminal-behavioral model (TBM)

\section{DC-DC Converter}

An example of $d c-d c$ converter terminal-behavioral modeling and decoupling procedure has been already shown in [6], and will not be repeated here. Both verification using average models and the validation through the experiment have been presented there.

\section{CONCLUSION}

The paper addresses un-terminated low-frequency terminal-behavioral modeling. Decoupling procedure has been shown in details and was verified on a few simulation examples using switching and average models. The results obtained using model in a frequency domain match well with the results obtained using average or switching models if the converter/system is dominantly linear. It has to be mentioned that this terminal modeling (and decoupling) can be applied to the much more complex systems: arbitrary converter from the system can be identified on-line, without a need to be taken out and characterized. The only constrain that system has to satisfy when obtaining the frequency response functions in practice is that steady-state operating conditions should not significantly change during the time required to perform small-signal measurements. Future work will certainly be oriented towards the validation of the un-terminated modeling and decoupling procedure through experiments.

\section{REFERENCES}

[1] J.A. Rosero, J.A. Ortega, E. Aldabas, L. Romeral, "Moving towards a more electric aircraft" Aerospace and Electronic Systems Magazine, IEEE Volume 22, Issue 3, March 2007.

[2] T. J. McCoy and J. V. Amy, "The state-of-the-art of integrated electric power and propulsion systems and technologies on ships," Proc. IEEE ESTS, pp. 340-344, Apr. 2009.

[3] A. Emadi, Y. J. Lee, K. Rajashekara, "Power electronics and motor drives in electric, hybrid electric, and plug-in hybrid electric ve- hicles," IEEE Trans. Industrial Electron., vol. 55, no. 6, pp. 22372245, Sep. 2008.

[4] D. Izquierdo, R. Azcona, F. del Cerro, C. Fernandez, and B. Delicado, "Electrical power distribution system (HV270DC), for application in more electric aircraft," in Applied Power Electronics Conference and Exposition (APEC), 2010 Twenty-Fifth Annual IEEE, 2010, pp. 1300-1305.

[5] D. Boroyevich, I. Cvetkovic, D. Dong, R. Burgos, W. Fei, and F. Lee, "Future electronic power distribution systems a contemplative view," in Optimization of Electrical and Electronic Equipment (OPTIM), 2010 12th International Conference on, 2010, pp. 1369-1380.

[6] I. Cvetkovic, D. Boroyevich, P. Mattavelli, F. C. Lee, and D. Dong, "Un-terminated, low-frequency terminal behavioral model of dc-dc converters," in Applied Power Electronics Conference and Exposition (APEC), 2011 Twenty-Sixth Annual IEEE, 2011, pp. 1873-1880.

[7] L. Arnedo, R. Burgos, F. Wang, and D. Boroyevich, "Black-box terminal characterization modeling of dc-to-dc converters," IEEE APEC '07, pp. 457-463, Feb. 2007.

[8] J. A. Oliver, R. Prieto, V. Romero, and J. A. Cobos, "Behavioral modeling of $\mathrm{dc}-\mathrm{dc}$ converters for large-signal simulation of distributed power systems," in Applied Power Electronics Conference and Exposition, 2006. APEC '06. Twenty-First Annual IEEE, 2006.

[9] L. Arnedo, D. Boroyevich, R. Burgos, and F. Wang, "Un-terminated frequency response measurements and model order reduction for black-box terminal characterization models," in Applied Power Electronics Conference and Exposition, 2008. APEC 2008. Twenty-Third Annual IEEE, 2008, pp. 1054-1060.

[10] V. Valdivia, A. Barrado, A. Lazaro, C. Fernandez, and P. Zumel, "Black-box modeling of DC-DC converters based on transient response analysis and parametric identification methods," in Applied Power Electronics Conference and Exposition (APEC), 2010 TwentyFifth Annual IEEE, 2010, pp. 1131-1138.

[11] J. A. Oliver, R. Prieto, J. A. Cobos, O. Garcia, and P. Alou, "Hybrid Wiener-Hammerstein Structure for Grey-Box Modeling of DC-DC Converters," in Applied Power Electronics Conference and Exposition, 2009. APEC 2009. Twenty-Fourth Annual IEEE, 2009, pp. 280285.

[12] I. Cvetkovic, D. Boroyevich, P. Mattavelli, F. C. Lee, and D. Dong, "Non-linear, hybrid terminal behavioral modeling of a dc-based nanogrid system," in Applied Power Electronics Conference and Exposition (APEC), 2011 Twenty-Sixth Annual IEEE, 2011, pp. 12511258.

[13] Bishnoi, A. C. Baisden, P. Mattavelli, and D. Boroyevich, "EMI modeling of half-bridge inverter using a generalized terminal model," in Applied Power Electronics Conference and Exposition (APEC), 2011 Twenty-Sixth Annual IEEE, 2011, pp. 468-474.

[14] A. C. Baisden, D. Boroyevich, and F. Wang, "EMI Terminal Modeling," in Industry Applications Society Annual Meeting, 2008. IAS '08. IEEE, 2008, pp. 1-8.

[15] Y. A. Familiant, H. Jing, K. A. Corzine, and M. Belkhayat, "New Techniques for Measuring Impedance Characteristics of Three-Phase AC Power Systems," Power Electronics, IEEE Transactions on, vol. 24, pp. 1802-1810, 2009.

[16] V. Valdivia, A. Lazaro, A. Barrado, P. Zumel, C. Fernandez, and M. Sanz, "Impedance Identification Procedure of Three-Phase Balanced Voltage Source Inverters Based on Transient Response Measurements," Power Electronics, IEEE Transactions on, vol. PP, pp. 1-1, 2011.

[17] H. Jing, K. A. Corzine, and M. Belkhayat, "Small-Signal Impedance Measurement of Power-Electronics-Based AC Power Systems Using Line-to-Line Current Injection," Power Electronics, IEEE Transactions on, vol. 24, pp. 445-455, 2009.

[18] D. Boroyevich, R. Burgos, L. Arnedo, and W. Fei, "Synthesis and Integration of Future Electronic Power Distribution Systems," in Power Conversion Conference - Nagoya, 2007. PCC '07, 2007, pp. $\mathrm{K}-1-\mathrm{K}-8$.

[19] S. Jian, "Small-Signal Methods for AC Distributed Power Systems A Review," Power Electronics, IEEE Transactions on, vol. 24, pp. 2545-2554, 2009. 